\title{
Los usuarios de las bibliotecas y centros de documentación opinan sobre el funcionamiento de sus servicios durante la pandemia de Covid-19
}

\author{
Entrevista realizada por BLANCA SAN JOSÉ MONTANO, ELENA LÓPEZ DE LA FUENTE Y ANA \\ NASEIRO RAMUDO \\ Vocalía de Publicaciones de SEDIC
}

\begin{abstract}
Se realiza una entrevista a varios usuarios de bibliotecas y centros de documentación para conocer su experiencia, incertidumbres y deseos acerca del acceso a los servicios durante el estado de alarma de la Covid-19. Su experiencia ha sido positiva, con las bibliotecas funcionando y activas, en continua comunicación con sus usuarios. Valoran la gran profesionalidad del colectivo bibliotecario y su adaptabilidad a la situación de alarma y desescalada.
\end{abstract}

Biblioteca, Centros de documentación, Covid-19, Pandemia, Usuarios, Opinión, Servicios

\section{Introducción}

La situación generada a raíz de la pandemia durante el presente año, ha supuesto un cambio significativo en los servicios de las bibliotecas y centros de documentación; los principales afectados e interesados de estos cambios han sido los usuarios, cuyas necesidades son las razones de existir de nuestras instituciones. Los profesionales de la información y la documentación han hecho grandes esfuerzos para no bajar el nivel de satisfacción de sus usuarios, reinventándose con nuevos servicios y vías de comunicación, mejorando los sistemas de acceso a la información, ampliando la disponibilidad de recursos digitales documentales y bibliográficos. Los usuarios también han experimentado cambios en sus relaciones con los centros a los que habitualmente acuden en la búsqueda de orientación informativa y bibliográfica. Por esa razón, para conocer su experiencia, incertidumbres y deseos respecto a sus servicios, hemos entrevistado a 6 usuarios de diferente tipología de Instituciones.

Bajo el principio de que el acceso abierto a los resultados de investigación acelera el avance del conocimiento, todos los contenidos de la edición electrónica de CLIP se distribuyen bajo una licencia de uso y distribución Creative Commons Reconocimiento-NoComercialCompartirlgual 3.0 España (CC BY-NC-SA 3.0 ES). 


\section{Cuestionario}

1. ¿Cómo ha sido su experiencia en el uso de Biblioteca durante el estado de alarma y la desescalada? ¿qué ha echado de menos: la asistencia a la sala de consulta, algún servicio concreto, el trato con el personal...?

Pilar Gato Roca (Usuaria de biblioteca escolar)

Durante el proceso de confinamiento, solamente hemos tenido oportunidad de realizar como tutores de las distintas clases el servicio de préstamo y conocer las novedades bibliográficas que ofrecía la biblioteca escolar. Al no haber clases presenciales, todos los servicios que anteriormente ofrecía la biblioteca se vieron reducidos.

Guadalupe Jiménez (Usuaria de biblioteca especializada de ciencias de la salud)

Ha resultado una muy buena experiencia. A pesar de la distancia física impuesta por la pandemia, el esfuerzo de los profesionales de la biblioteca ha sido en todo momento encomiable para que tuviéramos todas nuestras necesidades de información cubiertas.

Gonzalo Salazar Mardones (Usuario de biblioteca universitaria)

La experiencia, como siempre muy positiva; el acceso a las publicaciones electrónicas, a pesar de la elevada saturación de la red universitaria, ha funcionado perfectamente pudiendo acceder tanto a las bases de datos de referencias bibliográficas como a las revistas científicas, directamente, desde los enlaces de la biblioteca.

En mi caso, mis accesos son sobre todo electrónicos y no se han visto afectados, pero los estudiantes de la Facultad con los que mantuve un contacto continuo durante la pandemia, echaban de menos la posibilidad de consulta en sala durante el confinamiento, a pesar de tener ya un gran número de manuales y libros en formato electrónico.

Francisco de Paula Roig Guerrero (Usuario de biblioteca especializada en economía)

Mi experiencia en general ha sido muy positiva. Lógicamente, al no poder acudir en persona, no he podido consultar material en formato papel, pero ello ha sido producto de las circunstancias y no una deficiencia del servicio, que ha continuado prestándose con su nivel acostumbrado. Sí que he echado en falta el contacto en persona con los compañeros de la biblioteca, pues éste siempre da pie a conversaciones en los que surgen ideas o sugerencias espontáneas que me ayudan a conocer mejor la biblioteca, sus novedades o sugerencias de libros.

Dicho lo anterior, gran cantidad del material que consultaba con anterioridad a la crisis sanitaria (artículos académicos, publicaciones especializadas, bases de datos...) ya accedía al mismo en formato electrónico, por lo que mi acceso al mismo no se ha visto alterado al tener ya configurado en mi ordenador de casa el acceso a estos recursos.

Varios usuarios de bibliotecas públicas

- Al principio fue duro no poder venir.

- Buenas. Manejaron muy bien el tema de la cita previa.

- No he venido 
- Echo de menos a la bibliotecaria.

- El cine-forum y los clubes de lectura. Estar con mis amigas del club de lectura.

\section{2. ¿Cómo ha sido la comunicación con el organismo y con sus profesionales?}

Pilar Gato Roca (Usuaria de biblioteca escolar)

Todas las comunicaciones se realizaron online. No obstante, y aparte de las dificultades, la biblioteca escolar ha respondido positivamente ante cualquier solicitud por parte del profesorado.

Guadalupe Jiménez (Usuaria de biblioteca especializada de ciencias de la salud)

La comunicación ha sido excelente en todo momento.

Gonzalo Salazar Mardones (Usuario de biblioteca universitaria)

Ante los problemas que me transmitían los estudiantes, normalmente de acceso desde sus domicilios a los fondos bibliográficos electrónicos, les remitía a los profesionales de la Biblioteca. Casi a la vuelta de correo electrónico, los estudiantes me indicaban que ya les habían atendido y solucionado los problemas, pudiendo acceder ya a los fondos electrónicos. Por mi parte, alguna duda sobre la incorporación de nuevos investigadores de la Universidad de Burgos a los servicios de la Biblioteca me fue rápidamente atendida y solventada por teléfono, y por la remisión inmediata de los formularios para su cumplimentación a través del correo electrónico.

Es decir, en todos los casos la comunicación ha sido fluida, eficaz y muy rápida.

Francisco de Paula Roig Guerrero (Usuario de biblioteca especializada en economía)

Muy fluida. Como comentaba en la anterior respuesta, el nivel de servicio ha continuado con el nivel que tenía antes de la crisis sanitaria, si bien limitado a la vía telemática. En este sentido, además del email, la plataforma de comunicación Teams ha resultado un buen sustituto del teléfono de trabajo que no tenemos en el domicilio particular, permitiendo además la videollamada.

Usuarios de bibliotecas públicas

- Muy bien. Todos muy amables.

- Buena.

- Excelente. Siempre me ayudan.

3. ¿Cómo valora el plan de desescalada que han utilizado? ¿Lo conoce? ¿Le parece efectivo y seguro?

Pilar Gato Roca (Usuaria de biblioteca escolar) 
Son muchas las limitaciones que conlleva la nueva normalidad y la biblioteca escolar es uno de los espacios más desfavorecidos, teniendo que limitar sus funciones en un grado sumo.

Guadalupe Jiménez (Usuaria de biblioteca especializada en ciencias de la salud)

Pusieron normas de acceso y uso acordes a las necesidades del momento

Gonzalo Salazar Mardones (Usuario de biblioteca universitaria)

A pesar de que los estudiantes no se incorporaron en la Universidad de Burgos a la docencia presencial, en el plan de desescalada pudieron acceder - previa solicitud telemática- a recoger libros que no se encontraban en versión electrónica. Esto les ha permitido complementar sus apuntes y la documentación referenciada por los profesores.

Como investigador, la posibilidad de devolver libros en la desescalada me permitió no acumularlos durante el verano, por si algún otro usuario los necesitara.

Francisco de Paula Roig Guerrero (Usuario de biblioteca especializada)

Debido a los rebrotes, no conozco que mi institución haya aplicado un plan de desescalada, pues no ha habido una vuelta al trabajo presencial desde marzo de este año.

Usuarios de bibliotecas públicas

- En la evaluación que hemos realizado los usuarios lo valoran el plan con un 5 de media. Pero consideran que nada es seguro y que sí conocen el plan de desescalada (Bibliotecario).

- Bien.

- Sí, me parece fiable y me da seguridad.

\section{Ha tenido algún problema especial con el uso de la Biblioteca? ¿Cree que podría haberse evitado?}

Pilar Gato Roca (Usuaria de biblioteca escolar)

Dado el estado en que nos encontramos, la biblioteca escolar es uno de los espacios de los que tenemos que prescindir en muchos de los aspectos a los que estábamos acostumbrados a utilizar. Nuestro alumnado no puede disfrutar de él como era habitual, teniendo que prescindir de sesiones de lectura colectiva, comentarios de libros en el sentido de "club de lectura" por la reducción del espacio físico, participación en talleres literarios, etc.

Debido a los protocolos derivados de la pandemia, no creo que sea posible evitar las consecuencias de un funcionamiento reducido de este servicio. Esperemos nuevos tiempos.

Guadalupe Jiménez (Usuaria de biblioteca especializada en ciencias de la salud)

En mi caso no he tenido ningún problema y he podido acceder a la biblioteca física y virtual cuando lo he necesitado. 


\section{Gonzalo Salazar Mardones (Usuario de biblioteca universitaria)}

Como indicaba anteriormente, como investigador no he tenido ningún problema en los accesos telemáticos a información. Los alumnos tampoco me han referido sino cuestiones puntuales que les han quedado perfectamente solventadas y que aparecían explicadas en los magníficos manuales e información que la Biblioteca pone a su disposición. El problema relativo a la imposibilidad de acceder a los libros en el periodo de confinamiento, nos venía dado por las condiciones del estado de alarma, por lo que era imposible de subsanar. En cuanto se relajaron las medidas de confinamiento, la biblioteca arrancó con los servicios de acceso a fondos y préstamo, con las máximas medidas de protección y seguridad.

\section{Francisco de Paula Roig Guerrero (Usuario de biblioteca especializada en economía)}

Más que un problema, he echado en falta pequeñas mejoras incrementales para adaptarnos al trabajo a distancia, como arbitrar algún sistema para hacer llegar al domicilio libros que se precisen en el trabajo (desconozco si la biblioteca tenía arbitrado un sistema en este sentido). La biblioteca de la CNMC ha trabajado en este sentido adoptando una plataforma de préstamo de libros digitales, supliendo la falta de acceso al libro en papel. Como he trasladado a los compañeros de biblioteca, sería deseable que el acceso a estos libros digitales en préstamo fuera factible no solo en los ordenadores portátiles corporativos, sino también en las tabletas particulares, para una lectura más cómoda, y permitir el mayor número de formatos de archivo en los que se encuentre el libro digital (en especial, el formato ePub).

Usuarios de bibliotecas públicas.

- No, no tengo ningún problema.

- Para mí la biblioteca es como mi segunda casa.

\section{5. ¿Cuál ha sido el servicio que más ha valorado de la Biblioteca al que ha podido acceder durante el confinamiento y le desescalada? ¿Es un servicio nuevo?}

\section{Pilar Gato Roca (Usuaria de biblioteca escolar)}

El servicio que más he valorado fue la continua información sobre el material bibliográfico de nueva adquisición y la posibilidad de contar con todo aquel necesario para el desarrollo de mis actividades docentes.

Guadalupe Jiménez (Usuaria de biblioteca especializada en ciencias de la Salud):

Poder acceder desde casa a la biblioteca virtual porque en el trabajo no teníamos tiempo. En cuanto a los servicios nuevos ha sido interesante el portal que crearon con artículos y documentos COVID19 ESPECIALIDADES.

Gonzalo Salazar Mardones (Usuario de biblioteca universitaria) 
La renovación automática del préstamo de libros durante el periodo de confinamiento y desescalada me ha parecido una gran idea que ha evitado preocupaciones a alumnos y profesores relativos a la necesidad de devolución de los ejemplares. El préstamo de portátiles, si bien fue suplementado con componentes desde el Vicerrectorado de Infraestructuras, ha tenido una importancia propia en este período al permitir acceder a la docencia telemática a algunos de los estudiantes que normalmente usaban medios propios menos adecuados (teléfonos) ya que anteriormente únicamente realizaban accesos puntuales a las plataformas digitales. La actualización de noticias sobre el funcionamiento de los servicios, la notificación de accesos abiertos a fondos durante el confinamiento, o la incorporación de nuevas colecciones ha sido muy de agradecer ya que nos ha permitido a investigadores y estudiantes conocer el estado actualizado de los servicios de la Biblioteca.

Francisco de Paula Roig Guerrero (Usuario de biblioteca especializada en economía)

Sin duda, el mejor servicio es haber tenido instalado en mi ordenador portátil el acceso automático (vía software de introducción automática de credenciales) a todos los recursos electrónicos con suscripción disponibles en la biblioteca digital de la CNMC. Este acceso lo tenía ya configurado en mi ordenador de la oficina y he podido seguir utilizándolo desde mi domicilio particular.

Usuarios de bibliotecas públicas

- Los cuentos por Youtube.

- eBiblioMadrid.

- He utilizado la página Web de la biblioteca y el Portal del lector para hacer listados para leer y recogerlos en préstamos.

6. ¿Cree que se ha mantenido la tradicional profesionalidad del personal de Bibliotecas con la actual situación, o piensa que se han superado sus expectativas?

Pilar Gato Roca (Usuaria de biblioteca escolar)

A todos/as nos sorprendió la situación, primero de confinamiento, después de una nueva normalidad incierta. La biblioteca escolar es uno de los espacios educativos que más ha sufrido esta situación. Esperamos que poco a poco se pueda ir solventando y que vuelva a servir de vínculo necesario para coordinar el proceso lecto-escritor del centro con todas las consecuencias. De todas formas, el personal que lleva el Equipo de Dinamización de la Biblioteca Escolar está activo y dispuesto a su servicio como es habitual.

Guadalupe Jiménez (Usuaria de biblioteca especializada en ciencias de la salud)

Sí, han seguido siendo muy profesionales, como siempre.

Gonzalo Salazar Mardones (Usuario de biblioteca universitaria)

La profesionalidad del personal ha quedado reflejada en su trabajo diario desde sus domicilios en el período de confinamiento. El servicio se ha mantenido perfectamente -al menos en lo que respecta a los servicios que personalmente empleo- no apreciándose ningún menoscabo en la calidad o 
rapidez de respuesta frente a las pequeñas dudas o cuestiones que se les planteaba. Como decía anteriormente, me consta que los estudiantes son de esa misma opinión ya que me han trasladado su satisfacción con los servicios que ellos han empleado.

\section{Francisco de Paula Roig Guerrero (Usuario de biblioteca especializada en economía)}

En mi opinión, el personal de biblioteca de la CNMC ha mantenido su profesionalidad en la actual situación y ha puesto un empeño especial en que el nivel de servicio no decayera por la imposibilidad de acudir presencialmente a la biblioteca. Además, como comentaba anteriormente, ha tratado de mejorar la experiencia del usuario de la biblioteca a distancia incorporando como servicio una plataforma de préstamo de libros en formato electrónico.

\section{Usuarios de bibliotecas públicas}

- Se han mantenido muy profesionales y han mantenido las normas.

- Se han adaptado muy bien a la circunstancia.

- Bien.

\section{7. ¿Cuál es su visión del funcionamiento de la biblioteca/archivo en un futuro cercano: servicios, espacios, profesionales...?}

\section{Pilar Gato Roca (Usuaria de biblioteca escolar)}

Todo depende de cómo se vayan sucediendo los acontecimientos respecto a la pandemia. No dudo en absoluto que su adaptación a los nuevos avatares sea positiva.

Guadalupe Jiménez (Usuaria de biblioteca especializada en ciencias de la salud)

Que deben adaptarse a las nuevas especialidades y tener más documentos tipo vídeos, tutoriales, canales, etc. ,que nos ayuden en momentos como este.

Gonzalo Salazar Mardones (Usuario de biblioteca universitaria)

Esta pandemia nos debe servir para ser capaces de hacer una apuesta decidida por el teletrabajo de los profesionales que pueden manejar las colecciones, compras, catalogación, etc., en remoto, siempre que se trate de contenidos digitales.

Asimismo, la apuesta por este tipo de fondos digitales disminuye/mantiene las necesidades de espacio pero, por el contrario, necesita de una mayor y más actualizada infraestructura informática tanto de software como de hardware que permita manejar un gran volumen de accesos (como ha sido el caso en el confinamiento) con la suficiente velocidad. Por ello, entiendo el futuro con una mayor compra de libros/manuales para los estudiantes en formato electrónico, al ser ellos nativos digitales que consultan los fondos desde sus propios terminales.

Del mismo modo debe potenciarse (licencias, cursos de formación, etc.), tanto para estudiantes como para profesores, el uso de gestores electrónicos de bases de datos, o el manejo de los software específicos de préstamo electrónico de libros. 
En relación con las bases de datos de referencias bibliográficas o con las revistas científicas electrónicas, puedo decir que la disponibilidad en la Universidad de Burgos es ejemplar, y el futuro pasa únicamente por mantener esa apuesta que ya se realizó hace muchos años, y que hace nuestra biblioteca como un ejemplo de apoyo a los investigadores y docentes.

\section{Francisco de Paula Roig Guerrero (Usuario de biblioteca especializada)}

Creo que el futuro del funcionamiento pasa por reforzar el acceso digital a los contenidos que precise el usuario. En este sentido, las ediciones en formato digital de libros que antes solo se adquirían en formato papel se van a extender. Asimismo, la posibilidad de poder acceder a los libros en múltiples dispositivos (ordenadores corporativos) sería deseable.

Otra faceta que creo que se podría explorar con el teletrabajo es la impartición de talleres o cursos de formación sobre los recursos de la biblioteca (digitales o no) en plataformas telemáticas como Teams (que es la que se emplea en la CNMC).

\section{Usuarios de bibliotecas públicas}

- No tengo una visión muy clara, con que siga funcionando me parece bien.

- Un poco más de talleres grupales.

- Que siga.

\section{8. ¿Le gustaría añadir algo?}

\section{Pilar Gato Roca (Usuaria de biblioteca escolar)}

Solamente, que la biblioteca escolar del centro está preparada para solventar las dificultades de esta situación, dado que su equipo de apoyo cuenta con profesionales perfectamente aptos para abordar los problemas derivados de la misma, reorganizando su funcionamiento con el fin de seguir prestando el servicio esencial habitual.

\section{Gonzalo Salazar Mardones (Usuario de biblioteca universitaria)}

La capacidad de adaptación del personal de la Biblioteca de la Universidad de Burgos al estado de confinamiento ha sido ejemplar permitiendo mantener un servicio excelente a estudiantes, profesores e investigadores. Asimismo, la gestión del servicio establecida desde su dirección y desde el Rectorado de la Universidad, ha permitido un mantenimiento de todos los servicios - a excepción lógicamente de los estrictamente presenciales-, adaptándose a los estados de desescalada incrementando sus servicios y recuperando "en lo posible" la normalidad y el servicio a los investigadores que se fueron incorporando en las distintas fases de esa desescalada.

Francisco de Paula Roig Guerrero (Usuario de biblioteca especializada en economía)

En anteriores preguntas ya he señalado todos los aspectos que me han parecido dignos de mención.

Usuarios de bibliotecas públicas 
- Gracias.

- Excelente servicio.

- Echo de menos pasar más tiempo en la zona infantil, las actividades (cuentacuentos, talleres...).

Podemos decir que la experiencia de los usuarios es positiva, las bibliotecas han seguido funcionando de forma muy activa, dando acceso a sus fondos y servicios, muy especialmente las universitarias, las de ciencias de la salud y las especializadas en economía, que ya tenían muy desarrollados servicios a distancia y colecciones electrónicas. Y las más perjudicadas han sido las bibliotecas escolares y las públicas que tienen unos servicios más presenciales (club de lectura, lectura colectiva...); además, la biblioteca pública tiene un gran valor como espacio de socialización que sus usuarios echan de menos.

En todas ellas se ha mantenido la comunicación con el usuario de forma fluida y eficaz online, pero echan de menos la presencialidad.

Los servicios más valorados son el acceso a los fondos, la continua información actualizada que les facilitan, la renovación automática de los préstamos y los modos alternativos en línea de los servicios tradicionales.

Todos los usuarios han valorado la gran profesionalidad del colectivo bibliotecario que se han mantenido trabajando, a pesar de los problemas, desde sus domicilios o, según los casos, en las instalaciones de las bibliotecas cuando se inició la desescalada.

Por último, los usuarios ven un futuro inmediato los fondos cada vez más digitales, como los servicios y más uso de canales de comunicación a distancia.

\section{Usuarios consultados}

- Pilar Gato Roca. Profesor de Primera del Colegio Mato Vizoso de Vilalba, Lugo.

- Dra. Guadalupe Jiménez. Oftalmología. Hospital San Pedro de Alcántara. Cáceres.

- Gonzalo Salazar Mardones. Profesor del Área de Ingeniería Química Decano de la Facultad de Ciencias. Universidad de Burgos.

- Francisco de Paula Roig Guerrero, Inspector Jefe de Competencia, Comisión Nacional de los Mercados y la Competencia (CNMC).

- Usuarios de las bibliotecas públicas de la Comunidad Autónoma de Madrid. 